Теорія Ймовір. та Матем. Статист. Вип. 78, 2008
Theor. Probability and Math. Statist. No. 78, 2009, Pages 175-190 S 0094-9000(09)00771-6

Article electronically published on August 4, 2009

\title{
MINIMUM VARIANCE HEDGING IN A MODEL WITH JUMPS AT POISSON RANDOM TIMES
}

UDC 519.21

\author{
V. M. RADCHENKO
}

\begin{abstract}
We consider a model where the price of an option is driven by a Wiener process and changes randomly at the moments determined by a homogeneous Poisson process. The formula for the minimum variance hedging strategy is obtained for a European type call option. The derivation of the formula is based on the FöllmerSchweizer decomposition of a contingent claim.
\end{abstract}

\section{INTRODUCTION}

In this paper, we consider the problem of finding a hedging self-financing strategy for a European type call option with the exercise time $T>0$ for a one dimensional model with continuous time. We assume that the discounted price of a stock $\left(X_{t}\right)_{0 \leq t \leq T}$ is driven by a Wiener process and changes randomly at the moments determined by a homogeneous Poisson process. The result of applying the minimum variance hedging strategy is the best approximation of the contingent claim in the mean square sense.

A contingent claim $H$ is a square integrable random variable satisfying a standard condition of integrability. Generally speaking, the market considered in the model below is incomplete. The contingent claim is not necessarily attainable. For a given $H$, we solve the following problem:

$$
\text { minimize } \mathrm{E}\left[H-V_{0}-\int_{0}^{T} \vartheta_{s} d X_{s}\right]^{2} \quad \text { over all strategies } \vartheta \text { and } V_{0} \in \mathbb{R} \text {. }
$$

If $\vartheta^{H}$ and $V_{0}^{H}$ are solutions of the above problem, then $\vartheta^{H}$ is called the minimum variance hedging strategy and $V_{0}^{H}$ is called the price of the contingent claim $H$.

In the model under consideration, $X$ is a special discontinuous semimartingale. The solution of problem (1.1) for semimartingales satisfying certain additional conditions is obtained in [1] for the general case. Some further results in this direction can be found in [2] and [3]. The hedging strategy in [1] is found as a solution of a stochastic equation whose elements are found from the Föllmer-Schweizer decomposition

$$
H=H_{0}+\int_{0}^{T} \xi_{s}^{H} d X_{s}+L_{T}^{H}
$$

where $H_{0} \in \mathbb{R}$ and $L^{H}$ is a martingale that is orthogonal to the martingale component of $X$. Nevertheless the problem of finding $\xi_{s}^{H}$ is not yet solved in the general case. A

2000 Mathematics Subject Classification. Primary 91B28; Secondary $60 \mathrm{H} 05$.

Key words and phrases. Minimum variance hedging, European call option, Föllmer-Schweizer decomposition, option price model with jumps, minimal martingale measure.

Supported by the Alexander von Humboldt Foundation, Grant \#1074615. 
possible approach for finding $\xi_{s}^{H}$ is mentioned in [4] for the case of a one dimensional $X$ (see the proof of Theorem 10 and a remark to this theorem in [4).

We show below that the results of the paper [1] hold for our model, too. We find $H_{0}, \xi_{t}^{H}$, and $L_{t}^{H}$ and the solution of problem (1.1) for a contingent claim of the form $H=f\left(X_{T}\right)$, where $f: \mathbb{R} \rightarrow \mathbb{R}_{+}$is a continuous function whose growth is at most polynomial.

Starting with the classical paper 5 , various modifications of this model of the stock price are considered in a number of papers (see, for example, 6, Chapter 10] or [7. Section 7]), since it is a natural model of the financial market. However the explicit formulas for the mean square hedging strategy are obtained only for the case where the price of a stock is a martingale, that is, if the parameters of the stock price satisfy a special equality (see [7, Section 7.3.4] for the case of jumps driven by the Poisson process or [6, Section 10.4.2] for the case of an exponential Lévy process). In the case of the model considered below, we impose a special condition on the model in the form of an inequality (see inequality (2.3) below).

The formulas for the mean square hedging strategy were obtained recently in 8 for the case where $X$ is an exponential Lévy process. It is worthwhile mentioning that the contingent claim in 8 is of the form $H=f\left(X_{T}\right)$, where $f(s)=\int s^{z} \Pi(d z)$ and where the measure $\Pi$ is defined in the formula for the hedging strategy. The existence of such an integral representation is proved in 8 only for some special cases of $f$. Instead, we impose other conditions on $f$ and express the hedging strategy in different terms.

Following the method of the paper [1, we find the hedging strategy with the help of the conditional expectation with respect to the minimal martingale measure. Note that other approaches for finding the strategies for incomplete markets are based on the notions of the minimal entropy martingale measure [9] or minimal risk martingale measure [10, 11. If the moments of jumps are nonrandom and are known in advance, then the mean square hedging strategy is obtained in [12] explicitly.

In Section 2 we introduce the model of interest and give some necessary definitions and results. The Föllmer-Schweizer decomposition for the stock price in our model is obtained in Section 3. The formulas for the hedging strategy are derived from this decomposition in Section 4. The Appendix contains the proof of an auxiliary result.

\section{THE MODEL}

Let $\left(N_{t}\right)_{0 \leq t \leq T}$ be a homogeneous Poisson process with intensity $\lambda$ and denote by $\tau_{j}$, $j \geq 1$, the increasing sequence of moments when the process $N$ jumps. For convenience, we assume that $\tau_{0}=0$. Without loss of generality we also assume that $N_{T-}=N_{T}$.

Let $\left(W_{t}\right)_{0 \leq t \leq T}$ be a Wiener process and let $\left(U_{j}\right)_{j \geq 1}$ be independent identically distributed random variables assuming values in the interval $(-1,+\infty)$. We further assume that $\mathrm{E} U_{j}^{n}<\infty$ for all $n \geq 1$. Denote the distribution function of $U_{j}$ (which is independent of $j$ ) by $\nu$. We also put $E_{1}=\mathrm{E} U_{j}$ and $E_{2}=\mathrm{E} U_{j}^{2}$.

Let the $\sigma$-algebras $\sigma\left(N_{t}, 0 \leq t \leq T\right), \sigma\left(W_{t}, 0 \leq t \leq T\right)$, and $\sigma\left(U_{j}, j \geq 1\right)$ be independent. For every $0 \leq t \leq T$, denote by $\mathcal{F}_{t}$ the $\sigma$-algebra generated by all random variables $\left(W_{s}\right)_{0 \leq s \leq t},\left(N_{s}\right)_{0 \leq s \leq t}$, and $\left(U_{j}\right)_{j: 0<\tau_{j} \leq t}$ and completed with all sets of zero probability belonging to $\mathcal{F}$. We also assume that $\mathcal{F}=\mathcal{F}_{T}$.

We denote the discounted price of a stock (risky asset) at the moment $t$ by $X_{t}$ and assume that $X_{0} \in \mathbb{R}, X_{0}>0$. We tacitly assume that there exists a riskless asset (called a bond). Let the process $\left(X_{t}\right)_{0 \leq t \leq T}$ satisfy the equation

$$
X_{t}=X_{0}+\int_{0}^{t} X_{s-}\left(a d s+\sigma d W_{s}\right)+\sum_{j: 0<\tau_{j} \leq t} X_{\tau_{j}-} U_{j}, \quad 0 \leq t \leq T,
$$


where $a \geq 0$ and $\sigma>0$ are some constants. This implies that

$$
X_{t}=X_{0} \exp \left\{\left(a-\frac{\sigma^{2}}{2}\right) t+\sigma W_{t}\right\} \prod_{j: 0<\tau_{j} \leq t}\left(1+U_{j}\right), \quad 0 \leq t \leq T .
$$

Let

$$
\frac{a+E_{1} \lambda}{\sigma^{2}+E_{2} \lambda} U_{j}<1 \quad \text { a.s. }
$$

where "a.s." is the abbreviation for "almost surely". Below we show how important is the latter assumption.

In what follows we will apply the following assertion (its proof is given in Lemma 7.2.2 of the paper [7]).

Lemma 2.1. Let $\Phi(y, z): \mathbb{R}^{d} \times \mathbb{R} \rightarrow \mathbb{R}$ be a measurable function such that the function $y \mapsto \Phi(y, z)$ is continuous in $\mathbb{R}^{d}$ for all $z \in \mathbb{R}$ and let $\left(Y_{t}\right)_{0 \leq t \leq T}$ be a continuous from the left process assuming values in $\mathbb{R}^{d}$ and being adapted to the filtration $\left(\mathcal{F}_{t}\right)_{0 \leq t \leq T}$. Assume that

$$
\mathrm{E} \int_{0}^{T} d s \int_{\mathbb{R}} \nu(d z) \Phi^{2}\left(Y_{s}, z\right)<+\infty
$$

Then the process $\left(\tilde{M}_{t}\right)_{0 \leq t \leq T}$ defined by

$$
\tilde{M}_{t}=\sum_{j: 0<\tau_{j} \leq t} \Phi\left(Y_{\tau_{j}}, U_{j}\right)-\lambda \int_{0}^{t} d s \int_{\mathbb{R}} \nu(d z) \Phi\left(Y_{s}, z\right)
$$

is a square integrable martingale.

Note that $\left(X_{t}\right)_{0 \leq t \leq T}$ is a square integrable semimartingale in our model and moreover

$$
X_{t}=X_{0}+M_{t}+A_{t},
$$

where

$$
M_{t}=\sigma \int_{0}^{t} X_{s-} d W_{s}+\sum_{j: 0<\tau_{j} \leq t} X_{\tau_{j}-} U_{j}-E_{1} \lambda \int_{0}^{t} X_{s-} d s, \quad 0 \leq t \leq T,
$$

is a martingale (this conclusion follows from Lemma 2.1 for $\Phi(y, z)=y z$ and $Y_{t}=X_{t-}$ ) and

$$
A_{t}=\left(a+E_{1} \lambda\right) \int_{0}^{t} X_{s-} d s, \quad 0 \leq t \leq T,
$$

is a predictable process of bounded variation.

The bracket process associated to $M$ is obtained from (2.5) as follows:

$$
[M]_{t}=\sigma^{2} \int_{0}^{t} X_{s-}^{2} d s+\sum_{j: 0<\tau_{j} \leq t} X_{\tau_{j}-}^{2} U_{j}^{2} .
$$

This equality together with Lemma 2.1 (for $\Phi(y, z)=y^{2} z^{2}$ and $Y_{t}=X_{t-}$ ) yields the predictable bracket process, namely

$$
\langle M\rangle_{t}=\left(\sigma^{2}+E_{2} \lambda\right) \int_{0}^{t} X_{s-}^{2} d s .
$$

Put

$$
\rho=\frac{a+E_{1} \lambda}{\sigma^{2}+E_{2} \lambda} .
$$


Then

$$
\alpha_{t}=\frac{\rho}{X_{t-}}
$$

for $\alpha_{t}=d A_{t} / d\langle M\rangle_{t}$.

Let $\left(N_{t}^{(1)}\right)_{0 \leq t \leq T}$ and $\left(N_{t}^{(2)}\right)_{0 \leq t \leq T}$ be square integrable martingales such that

$$
N_{0}^{(1)}=N_{0}^{(2)}=0
$$

almost surely. Recall that $N^{(1)}$ and $N^{(2)}$ are called strongly orthogonal if

$$
\left(N_{t}^{(1)} N_{t}^{(2)}\right)_{0 \leq t \leq T}
$$

is a uniformly integrable martingale.

The following two definitions agree with the corresponding definitions in [1] and [13.

Definition 2.1. Denote the set of predictable processes $\left(\theta_{t}\right)_{0 \leq t \leq T}$ assuming values in $\mathbb{R}$ and such that $\left(\int_{0}^{t} \theta_{s}^{2} d\langle M\rangle_{s}\right)_{0 \leq t \leq T}$ is integrable by $L^{2}(M)$. We also denote the set of predictable processes $\left(\theta_{t}\right)_{0 \leq t \leq T}$ assuming values in $\mathbb{R}$ and such that $\left(\int_{0}^{t}\left|\theta_{s} \alpha_{s}\right| d\langle M\rangle_{s}\right)_{0 \leq t \leq T}$ is square integrable by $L^{2}(A)$. We also put $\Theta=L^{2}(M) \cap L^{2}(A)$.

A contingent claim $H$ is a square integrable and $\mathcal{F}_{T}$-measurable random variable.

Definition 2.2. Let $H$ be a random variable such that

$$
H \in L^{2}(\Omega, \mathcal{F}, \mathrm{P}) \text {. }
$$

The equality

$$
H=H_{0}+\int_{0}^{T} \xi_{s}^{H} d X_{s}+L_{T}^{H}
$$

is called the Föllmer-Schweizer decomposition of $H$, where $H_{0}$ is an $\mathcal{F}_{0}$-measurable random variable,

$$
\left(\xi_{t}^{H}\right)_{0 \leq t \leq T} \in \Theta, \quad L_{0}^{H}=0 \quad \text { a.s. }
$$

and $\left(L_{t}^{H}\right)_{0 \leq t \leq T}$ is a square integrable martingale such that $L_{t}^{H}$ and $\int \theta d M$ are strongly orthogonal for all $\theta \in L^{2}(M)$.

If the Föllmer-Schweizer decomposition (2.7) holds, then one can consider the intrinsic price process for $H$ given by

$$
V_{t}^{H}=H_{0}+\int_{0}^{t} \xi_{s}^{H} d X_{s}+L_{t}^{H}, \quad 0 \leq t \leq T .
$$

Then the process

$$
\hat{K}_{t}=\int_{0}^{t} \alpha_{s}^{2} d\langle M\rangle_{s}=\left(a+E_{1} \lambda\right) \rho t
$$

is bounded. Therefore condition (SC) of [1] holds, and Theorem 3.4 of 13 implies the existence and uniqueness of the Föllmer-Schweizer decomposition for any random variable $H \in L^{2}(\Omega, \mathcal{F}, \mathrm{P})$. Note that Lemma 2 in [1] implies that $\Theta=L^{2}(M)$.

The process

$$
\tilde{K}_{t}=\int_{0}^{t} \frac{\alpha_{s}^{2}}{1+\sum_{j: 0<s_{j} \leq s}\left(\mathrm{E} U_{j}\right)^{2}\left(\operatorname{Var} U_{j}\right)^{-1} I_{\left\{s_{j}\right\}}(s)} d\langle M\rangle_{s}=\hat{K}_{t}
$$


is deterministic, too. Thus all the assumptions of [1, Theorem 3 and Corollary 10] hold. It follows from Corollary 10 in [1] that the solution of the problem

$$
\text { minimize } \mathrm{E}\left[H-V_{0}-\int_{0}^{T} \vartheta_{s} d X_{s}\right]^{2} \text { over all }\left(V_{0}, \vartheta\right) \in \mathbb{R} \times \Theta
$$

is a pair $\left(H_{0}, \xi^{\left(H_{0}\right)}\right)$, where the strategy $\xi^{\left(H_{0}\right)}$ satisfies equation (2.2) [1. In this case, $H_{0}$ is called the price of a contingent claim $H$. We say that $\xi^{\left(H_{0}\right)}$ generates a self-financing minimal variance hedging strategy for a given $H$.

It is worthwhile mentioning that there are no exact formulas for elements of equation (2.2) in 11. Below we obtain the explicit formula for the solution of problem (2.9) in the framework of the model (2.1)-(2.2) under some restrictions imposed on $H$.

Note that the process $X$ in (2.1)-(2.2) is a martingale if $a+E_{1} \lambda=0$. The minimal variance hedging strategy is obtained in [7, Section 7.3.4] for this case.

We derive the elements of the Föllmer-Schweizer decomposition from the density of the minimal martingale measure. The following notion is introduced in [14].

Definition 2.3. The probability measure $\hat{P}$ is called the minimal martingale measure if

1) $\hat{P}$ is equivalent to $P$,

2) $d \hat{\mathrm{P}} / d \mathrm{P} \in L^{2}(\Omega, \mathcal{F}, \mathrm{P})$,

3) $\left(X_{t}\right)_{0 \leq t \leq T}$ is a martingale with respect to $\hat{\mathrm{P}}$,

4) every square integrable $\mathrm{P}$-martingale which is strongly orthogonal to $M$ with respect to $P$ is a martingale with respect to $\hat{P}$.

It follows from Theorem 8 in [15] that $\hat{P}$ is a unique solution of the following optimization problem:

$$
\operatorname{minimize} \int_{\Omega}(d \mathrm{Q} / d \mathrm{P}-1)^{2} d \mathrm{P}
$$

over all sign-changing local martingale measures $\mathrm{Q}$,

where $X$ is such that $\int_{\Omega}(d \mathrm{Q} / d \mathrm{P})^{2} d \mathrm{P}<\infty$

and where $X$ is given by (2.1). This implies that $\mathrm{Q}=\hat{\mathrm{P}}$ gives the minimal value of

$$
\int_{\Omega}(d \mathrm{Q} / d \mathrm{P})^{2} d \mathrm{P}
$$

over the set of measures $Q$ mentioned above. Thus $\hat{P}$ is the variance-optimal martingale measure in the terminology of the paper [16].

We consider the stochastic exponent

$$
\hat{Z}_{t}=\mathcal{E}\left(-\int_{0}^{t} \alpha_{s} d M_{s}\right)=\exp \left\{E_{1} \lambda \rho t-\frac{1}{2} \sigma^{2} \rho^{2} t-\sigma \rho W_{t}\right\} \prod_{j: 0<\tau_{j} \leq t}\left(1-\rho U_{j}\right) .
$$

Condition (2.3) implies that $\hat{Z}_{T}>0$ almost surely. According to Lemma 17 in [1], the measure $\hat{\mathrm{P}}$ defined by relation $d \hat{\mathrm{P}}=\hat{Z}_{T} d \mathrm{P}$ is the minimal martingale measure for the price process $X$. Throughout the paper we denote by $\hat{\mathrm{E}}$ the expectation with respect to the measure $\hat{P}$ whose density is given by (2.10). Note that the formula for the density of the minimal martingale measure for the model (2.1)-(2.2) is obtained in Proposition 2.1 of [17] (the formula in [17] is compatible with our result).

From Lemma 17 in [1], we get the following equalities for both the price $H_{0}$ and for the intrinsic price process $V_{t}^{H}$ :

$$
H_{0}=\hat{\mathrm{E}} H, \quad V_{t}^{H}=\hat{\mathrm{E}}\left(H \mid \mathcal{F}_{t}\right) .
$$


In particular, $\hat{\mathrm{P}}$ is an equivalent martingale measure for $X$. Therefore our model is arbitrage free (see, for example, Theorem VII.2a.2 in [18]).

We discuss briefly condition (2.3). If $-1 \leq \rho \leq 0$, then (2.3) holds for all

$$
U_{j} \in(-1,+\infty) \text {. }
$$

If $\rho>0$, then (2.3) implies that $U_{j}$ is bounded almost surely and condition $\mathrm{E} U_{j}^{n}<+\infty$ holds automatically.

\section{Derivation of the Föllmer-Schweizer decomposition}

In what follows we consider a contingent claim of the following form:

$$
H=f\left(X_{T}\right),
$$

where $f: \mathbb{R} \rightarrow \mathbb{R}_{+}$is a continuous function whose growth is at most polynomial. Note that $H \in L^{2}(\Omega, \mathcal{F}, \mathrm{P})$ under the above assumptions.

Considering equalities (2.7), (2.8), and (2.11), we try to obtain the decomposition

$$
V_{t}^{H}=H_{0}+\int_{0}^{t} \xi_{s}^{H} d X_{s}+L_{t}^{H}, \quad 0 \leq t \leq T,
$$

where $H_{0}, \xi^{H}$, and $L_{t}^{H}$ satisfy the conditions of Definition 2.2. In what follows we assume that $H$ is fixed and thus we usually omit the superscript $H$ for various variables. Let $\left(N_{t}\right),\left(W_{t}\right)$, and $\left(U_{j}\right)$ be defined on probability spaces $\Omega^{N}, \Omega^{W}$, and $\Omega^{U}$, respectively. The superscripts $N, W$, and $U$ indicate the expectations or elements in the corresponding probability spaces.

The random variables $\hat{Z}_{T} / \hat{Z}_{t}$ and $X_{t}$ are independent, whence

$$
V_{t}=\hat{\mathrm{E}}\left[f\left(X_{T}\right) \mid \mathcal{F}_{t}\right]=\mathrm{E}\left[f\left(X_{T}\right) \frac{\hat{Z}_{T}}{\hat{Z}_{t}} \mid \mathcal{F}_{t}\right]=F\left(t, X_{t}\right),
$$

where

$$
\begin{gathered}
F(t, x)=\mathrm{E}^{N} \bar{F}\left(t, x, \omega^{N}\right), \\
\bar{F}\left(t, x, \omega^{N}\right)=\mathrm{E}^{U} \mathrm{E}^{W}\left[f\left(x \frac{X_{T}}{X_{t}}\right) \frac{\hat{Z}_{T}}{\hat{Z}_{t}}\right] \\
=\mathrm{E}^{U} \mathrm{E}^{W}\left[f\left(x \exp \left\{\left(a-\frac{\sigma^{2}}{2}\right)(T-t)+\sigma\left(W_{T}-W_{t}\right)\right\} \prod_{j: t<\tau_{j}\left(\omega^{N}\right)<T}\left(1+U_{j}\right)\right)\right. \\
\times \exp \left\{E_{1} \lambda \rho(T-t)-\frac{1}{2} \sigma^{2} \rho^{2}(T-t)-\sigma \rho\left(W_{T}-W_{t}\right)\right\} \\
\omega^{N} \in \Omega^{N} .
\end{gathered}
$$

Since the function $f$ is continuous and its growth is at most polynomial, we obtain from the Lebesgue dominated convergence theorem that

$$
F(\cdot, x) \in \mathbb{C}[0, T]) .
$$

We have

$$
V_{t}=V_{0}+\sum_{j: 0<\tau_{j} \leq t}\left(V_{\tau_{j}-}-V_{\tau_{j-1}}\right)+\sum_{j: 0<\tau_{j} \leq t}\left(V_{\tau_{j}}-V_{\tau_{j}-}\right)+\left(V_{t}-V_{\tau_{N_{t}}}\right) .
$$


Consider the term

$$
V_{\tau_{j}-}-V_{\tau_{j-1}}=F\left(\tau_{j}-, X_{\tau_{j}-}\right)-F\left(\tau_{j-1}, X_{\tau_{j-1}}\right)
$$

For $1 \leq j \leq N_{T}+1, x \in \mathbb{R}$, and $\omega^{N} \in \Omega^{N}$, put

$$
g_{j}\left(x, \omega^{N}\right)=\mathrm{E}^{U}\left[f\left(x \prod_{i: j \leq i \leq N_{T}\left(\omega^{N}\right)}\left(1+U_{i}\right)\right) \prod_{i: j \leq i \leq N_{T}\left(\omega^{N}\right)}\left(1-\rho U_{i}\right)\right]
$$

(the products disappear if $j=N_{T}+1$, in which case $\left.g_{N_{T}+1}\left(x, \omega^{N}\right)=f(x)\right)$. Then the growth of functions $g_{j}\left(\cdot, \omega^{N}\right)$ is at most polynomial for all $\omega^{N} \in \Omega^{N}$.

Consider the functions

$$
\begin{aligned}
& \bar{F}_{j}\left(t, x, \omega^{N}\right)= \exp \left\{E_{1} \lambda \rho(T-t)\right\} \\
& \times \mathrm{E}^{W}\left[g_{j}\left(x \exp \left\{\left(a-\frac{\sigma^{2}}{2}\right)(T-t)+\sigma\left(W_{T}-W_{t}\right)\right\}, \omega^{N}\right)\right. \\
&\left.\quad \times \exp \left\{-\frac{1}{2} \sigma^{2} \rho^{2}(T-t)-\sigma \rho\left(W_{T}-W_{t}\right)\right\}\right]
\end{aligned}
$$

for $0 \leq t \leq T, x \in \mathbb{R}$, and $\omega^{N} \in \Omega^{N}$. Then

$$
\begin{aligned}
\bar{F}_{j}\left(t, x, \omega^{N}\right)= & \exp \left\{E_{1} \lambda \rho(T-t)\right\} \\
& \times \tilde{\mathrm{E}}^{W}\left[g_{j}\left(x \exp \left\{\left(a-\frac{\sigma^{2}}{2}\right)(T-t)+\sigma\left(W_{T}-W_{t}\right)\right\}, \omega^{N}\right)\right],
\end{aligned}
$$

where the expectation is considered in $\Omega^{W}$ with respect to a probability measure $\tilde{\mathrm{P}}^{W}$ such that

$$
\frac{d \tilde{\mathrm{P}}^{W}}{d \mathrm{P}^{W}}=\exp \left\{-\frac{1}{2} \sigma^{2} \rho^{2} T-\sigma \rho W_{T}\right\} .
$$

By the Girsanov theorem (see, for example, Corollary 13.25 and Theorem 13.27 in [19]) $\tilde{W}_{t}=W_{t}+\sigma \rho t$ is a Wiener process with respect to the probability measure $\tilde{\mathrm{P}}^{W}$. Hence

$$
\begin{aligned}
\bar{F}_{j}\left(t, x, \omega^{N}\right)=\exp \left\{E_{1} \lambda \rho(T-t)\right\} \tilde{\mathrm{E}}^{W} & {\left[g _ { j } \left(x \exp \left\{\left(a-\sigma^{2} \rho\right)(T-t)\right\}\right.\right.} \\
& \left.\left.\times \exp \left\{-\frac{\sigma^{2}}{2}(T-t)+\sigma\left(\tilde{W}_{T}-\tilde{W}_{t}\right)\right\}, \omega^{N}\right)\right] .
\end{aligned}
$$

Consider the function

$$
\begin{gathered}
\tilde{F}_{j}\left(t, y, \omega^{N}\right)=\tilde{\mathrm{E}}^{W}\left[g_{j}\left(y \exp \left\{-\frac{\sigma^{2}}{2}(T-t)+\sigma\left(\tilde{W}_{T}-\tilde{W}_{t}\right)\right\}, \omega^{N}\right)\right], \\
0 \leq t \leq T, \quad y \in \mathbb{R}, \quad \omega^{N} \in \Omega^{N} .
\end{gathered}
$$

It is known that the function $\tilde{F}_{j}\left(\cdot, \cdot, \omega^{N}\right) \in \mathbb{C}^{1,2}([0, T] \times \mathbb{R})$ is a solution of the equation

$$
\frac{\partial \tilde{F}_{j}}{\partial t}+\frac{1}{2} \sigma^{2} y^{2} \frac{\partial^{2} \tilde{F}_{j}}{\partial y^{2}}=0, \quad \tilde{F}_{j}\left(T, y, \omega^{N}\right)=g_{j}\left(y, \omega^{N}\right)
$$

for all $\omega^{N}$ (see, for example, Corollary 5.1.3 and equality (5.46) in [20] for $r=0$ ).

The function

$$
y(t, x)=x \exp \left\{\left(a-\sigma^{2} \rho\right)(T-t)\right\}
$$

is smooth in the case under consideration, whence

$$
\bar{F}_{j}\left(t, x, \omega^{N}\right)=\exp \left\{E_{1} \lambda \rho(T-t)\right\} \tilde{F}_{j}\left(t, y(t, x), \omega^{N}\right) \in \mathbb{C}^{1,2}([0, T] \times \mathbb{R})
$$


for all fixed $\omega^{N}$. Note that $\partial^{2} y / \partial x^{2}=0$, whence by using equations (3.2) we get

$$
\frac{\partial \bar{F}_{j}}{\partial t}+\frac{1}{2} \sigma^{2} x^{2} \frac{\partial^{2} \bar{F}_{j}}{\partial x^{2}}=\left(\sigma^{2} \rho-a\right) x \frac{\partial \bar{F}_{j}}{\partial x}-E_{1} \lambda \rho \bar{F}_{j} .
$$

Now

$$
\begin{array}{r}
\quad \begin{array}{l}
\bar{F}\left(t, x, \omega^{N}\right)=\bar{F}_{j}\left(t, x, \omega^{N}\right) \quad \text { for } t \in\left[\tau_{j-1}, \tau_{j}\right), \\
\bar{F}\left(t, x, \omega^{N}\right)=\bar{F}_{N_{T}+1}\left(t, x, \omega^{N}\right) \quad \text { for } t \geq \tau_{N_{T}},
\end{array} \quad x \in \mathbb{R}, \\
V_{\tau_{j-}-}-V_{\tau_{j-1}}=F\left(\tau_{j}-, X_{\tau_{j}-}\right)-F\left(\tau_{j-1}, X_{\tau_{j-1}}\right)
\end{array}
$$$$
\begin{aligned}
& =\left.\mathrm{E}^{N}\left[\bar{F}\left(c_{j}, y_{j}, \omega^{N}\right)-\bar{F}\left(b_{j}, x_{j}, \omega^{N}\right)\right]\right|_{b_{j}=\tau_{j-1}, c_{j}=\tau_{j}-, x_{j}=X_{\tau_{j-1}, y_{j}}=X_{\tau_{j}-}} \\
& =\mathrm{E}^{N}\left[\bar{F}\left(c_{j}, x_{j} \exp \left\{\left(a-\frac{\sigma^{2}}{2}\right)\left(c_{j}-b_{j}\right)+\sigma\left(W_{c_{j}}-W_{b_{j}}\right)\right\}, \omega^{N}\right)\right.
\end{aligned}
$$$$
\left.-\bar{F}\left(b_{j}, x_{j}, \omega^{N}\right)\right]\left.\right|_{\substack{b_{j}=\tau_{j-1}, c_{j}=\tau_{j}-\\ x_{j}=X_{\tau_{j-1}}}} .
$$

Put

$$
\begin{gathered}
\bar{X}_{s}^{(j)}=x_{j} \exp \left\{\left(a-\frac{\sigma^{2}}{2}\right)\left(s-b_{j}\right)+\sigma\left(W_{s}-W_{b_{j}}\right)\right\}, \\
\tau_{b}=\min \left\{\tau_{k}: \tau_{k}>b_{j}\right\}, \quad \tau_{c}=\max \left\{\tau_{k}: \tau_{k} \leq c_{j}\right\}
\end{gathered}
$$

for $x_{j}>0$ and $0 \leq b_{j} \leq s \leq c_{j} \leq T$. Since $\bar{F}_{j}$ is continuous, we can use $c_{j}$ (instead of $c_{j}$ ) in equalities (3.5) if $c_{j} \neq \tau_{c}$. This observation follows from equalities (3.4). Therefore

$$
\begin{aligned}
\bar{F}\left(c_{j},\right. & \left.x_{j} \exp \left\{\left(a-\frac{\sigma^{2}}{2}\right)\left(c_{j}-b_{j}\right)+\sigma\left(W_{c_{j}}-W_{b_{j}}\right)\right\}, \omega^{N}\right)-\bar{F}\left(b_{j}, x_{j}, \omega^{N}\right) \\
= & \bar{F}\left(c_{j}, \bar{X}_{c_{j}}^{(j)}, \omega^{N}\right)-\bar{F}\left(b_{j}, \bar{X}_{b_{j}}^{(j)}, \omega^{N}\right) \\
= & \bar{F}\left(c_{j}-, \bar{X}_{c_{j}}^{(j)}, \omega^{N}\right)-\bar{F}\left(\tau_{c}, \bar{X}_{\tau_{c}}^{(j)}, \omega^{N}\right) \\
& +\sum_{k: b_{j}<\tau_{k-1}, \tau_{k} \leq c_{j}}\left[\bar{F}\left(\tau_{k}-, \bar{X}_{\tau_{k}}^{(j)}, \omega^{N}\right)-\bar{F}\left(\tau_{k-1}, \bar{X}_{\tau_{k-1}}^{(j)}, \omega^{N}\right)\right] \\
& +\bar{F}\left(\tau_{b}-, \bar{X}_{\tau_{b}}^{(j)}, \omega^{N}\right)-\bar{F}\left(b_{j}, \bar{X}_{b_{j}}^{(j)}, \omega^{N}\right) \\
& +\sum_{k: b_{j}<\tau_{k} \leq c_{j}}\left[\bar{F}\left(\tau_{k}, \bar{X}_{\tau_{k}}^{(j)}, \omega^{N}\right)-\bar{F}\left(\tau_{k}-, \bar{X}_{\tau_{k}}^{(j)}, \omega^{N}\right)\right] .
\end{aligned}
$$

Using Itô's formula and making standard transformations we obtain

$$
\begin{aligned}
\bar{F}\left(\tau_{k}-\right. & \left., \bar{X}_{\tau_{k}}^{(j)}, \omega^{N}\right)-\bar{F}\left(\tau_{k-1}, \bar{X}_{\tau_{k-1}}^{(j)}, \omega^{N}\right) \\
= & \int_{\tau_{k-1}}^{\tau_{k}-} \frac{\partial \bar{F}}{\partial x}\left(s, \bar{X}_{s-}^{(j)}, \omega^{N}\right) d \bar{X}_{s}^{(j)} \\
& +\int_{\tau_{k-1}}^{\tau_{k}-}\left(\left(\sigma^{2} \rho-a\right) \bar{X}_{s-}^{(j)} \frac{\partial \bar{F}}{\partial x}\left(s, \bar{X}_{s-}^{(j)}, \omega^{N}\right)-E_{1} \lambda \rho \bar{F}\left(s, \bar{X}_{s-}^{(j)}, \omega^{N}\right)\right) d s
\end{aligned}
$$

by (3.3) and (3.4) for all fixed $\omega^{N} \in \Omega^{N}$ and $b_{j}<\tau_{k-1}, \tau_{k} \leq c_{j}$. The reasoning for the intervals $\left[b_{j}, \tau_{b}\right)$ and $\left[\tau_{c}, c_{j}\right)$ is similar. 
Now we derive from (3.6) and (3.7) that

$$
\begin{aligned}
\bar{F}\left(c_{j},\right. & \left.\bar{X}_{c_{j}}^{(j)}, \omega^{N}\right)-\bar{F}\left(b_{j}, \bar{X}_{b_{j}}^{(j)}, \omega^{N}\right) \\
= & \int_{b_{j}}^{c_{j}} \frac{\partial \bar{F}}{\partial x}\left(s, \bar{X}_{s-}^{(j)}, \omega^{N}\right) d \bar{X}_{s}^{(j)} \\
& +\int_{b_{j}}^{c_{j}}\left(\left(\sigma^{2} \rho-a\right) \bar{X}_{s-}^{(j)} \frac{\partial \bar{F}}{\partial x}\left(s, \bar{X}_{s-}^{(j)}, \omega^{N}\right)-E_{1} \lambda \rho \bar{F}\left(s, \bar{X}_{s-}^{(j)}, \omega^{N}\right)\right) d s \\
& +\sum_{k: b_{j}<\tau_{k} \leq c_{j}}\left[\bar{F}\left(\tau_{k}, \bar{X}_{\tau_{k}}^{(j)}, \omega^{N}\right)-\bar{F}\left(\tau_{k}-, \bar{X}_{\tau_{k}}^{(j)}, \omega^{N}\right)\right] .
\end{aligned}
$$

It is obvious that the expectations $\mathrm{E}^{N}$ of the integrals in (3.8) are equal to the integrals of expectations. It is also easy to prove that

$$
\begin{gathered}
\mathrm{E}^{N}\left(\frac{\partial \bar{F}}{\partial x}\left(s, \bar{X}_{s-}^{(j)}, \omega^{N}\right)\right) \bar{X}_{s-}^{(j)}=\frac{\partial F}{\partial x}\left(s, \bar{X}_{s-}^{(j)}\right) \bar{X}_{s-}^{(j)}, \\
\mathrm{E}^{N}\left(\left(\sigma^{2} \rho-a\right) \bar{X}_{s-}^{(j)} \frac{\partial \bar{F}}{\partial x}\left(s, \bar{X}_{s-}^{(j)}, \omega^{N}\right)-E_{1} \lambda \rho \bar{F}\left(s, \bar{X}_{s-}^{(j)}, \omega^{N}\right)\right) \\
=\left(\sigma^{2} \rho-a\right) \bar{X}_{s-}^{(j)} \frac{\partial F}{\partial x}\left(s, \bar{X}_{s-}^{(j)}\right)-E_{1} \lambda \rho F\left(s, \bar{X}_{s-}^{(j)}\right) .
\end{gathered}
$$

Recall that $\bar{X}_{s}^{(j)}$ depends on $x_{j}$. For $s, t \in\left[b_{j}, c_{j}\right], s \leq t$, put

$$
R\left(s, t, x_{j}\right)=\mathrm{E}^{N}\left(\sum_{k: s<\tau_{k} \leq t}\left[\bar{F}\left(\tau_{k}, \bar{X}_{\tau_{k}}^{(j)}, \omega^{N}\right)-\bar{F}\left(\tau_{k}-, \bar{X}_{\tau_{k}}^{(j)}, \omega^{N}\right)\right]\right)
$$

(if there is no term satisfying the restriction, then we agree that the sum equals zero). Considering the expectations $\mathrm{E}^{N}$ in (3.8) we have

$$
\begin{aligned}
F\left(c_{j}, \bar{X}_{c_{j}}^{(j)}\right)-F\left(b_{j}, \bar{X}_{b_{j}}^{(j)}\right) \\
=\int_{b_{j}}^{c_{j}} \frac{\partial F}{\partial x}\left(s, \bar{X}_{s-}^{(j)}\right) \bar{X}_{s-}^{(j)}\left(a d s+\sigma d W_{s}\right) \\
\quad+\int_{b_{j}}^{c_{j}}\left(\left(\sigma^{2} \rho-a\right) \bar{X}_{s-}^{(j)} \frac{\partial F}{\partial x}\left(s, \bar{X}_{s-}^{(j)}\right)-E_{1} \lambda \rho F\left(s, \bar{X}_{s-}^{(j)}\right)\right) d s+R\left(b_{j}, c_{j}, x_{j}\right) .
\end{aligned}
$$

Consider this equality for $x_{j}=X_{\tau_{j-1}}, b_{j}=\tau_{j-1}$, and $c_{j}=\tau_{j}-$. The stochastic integral is well defined for these variables, since they are independent of $d W_{s}$ on the intervals of interest. Thus we obtain

$$
\begin{aligned}
\sum_{j: 0<\tau_{j} \leq t} & \left(V_{\tau_{j}-}-V_{\tau_{j-1}}\right)+\left(V_{t}-V_{\tau_{N_{t}}}\right) \\
= & \int_{0}^{t} \frac{\partial F}{\partial x}\left(s, X_{s-}\right) X_{s-}\left(a d s+\sigma d W_{s}\right) \\
& +\int_{0}^{t}\left(\left(\sigma^{2} \rho-a\right) X_{s-} \frac{\partial F}{\partial x}\left(s, X_{s-}\right)-E_{1} \lambda \rho F\left(s, X_{s-}\right)\right) d s \\
& +\sum_{j: 0<\tau_{j} \leq t} R\left(\tau_{j-1}, \tau_{j}-, X_{\tau_{j-1}}\right)+R\left(\tau_{N_{t}}, t, X_{\tau_{N_{t}}}\right) .
\end{aligned}
$$

The process

$$
\sum_{j: 0<\tau_{j} \leq t} R\left(\tau_{j-1}, \tau_{j}-, X_{\tau_{j-1}}\right)+R\left(\tau_{N_{t}}, t, X_{\tau_{N_{t}}}\right)
$$


is continuous and has a bounded variation (moreover, this process is absolutely continuous; the proof of this result is given in the Appendix at the end of the paper). Thus the sign-changing measure generated by the increments of this process is absolutely continuous with respect to the Lebesgue measure.

For $0 \leq s \leq T$, we put

$$
\begin{gathered}
\psi_{s}=\frac{\partial F}{\partial x}\left(s, X_{s-}\right), \\
\varrho_{s}=\left(\sigma^{2} \rho-a\right) X_{s-} \frac{\partial F}{\partial x}\left(s, X_{s-}\right)-E_{1} \lambda \rho F\left(s, X_{s-}\right) \\
+\frac{d}{d s}\left(\sum_{j: 0<\tau_{j} \leq s} R\left(\tau_{j-1}, \tau_{j}-, X_{\tau_{j-1}}\right)+R\left(\tau_{N_{s}}, s, X_{\tau_{N_{s}}}\right)\right),
\end{gathered}
$$

where $R$ is defined by equality (3.9). Then

$$
\sum_{j: 0<\tau_{j} \leq t}\left(V_{\tau_{j}-}-V_{\tau_{j-1}}\right)+\left(V_{t}-V_{\tau_{N_{t}}}\right)=\int_{0}^{t} \psi_{s} X_{s-}\left(a d s+\sigma d W_{s}\right)+\int_{0}^{t} \varrho_{s} d s .
$$

Note that the process $\psi_{s}$ is left continuous and $\int_{0}^{t} \varrho_{s} d s$ is a continuous process of bounded variation.

We are going to derive the decomposition

$$
V_{t}=V_{0}+\int_{0}^{t} \xi_{s}^{H} X_{s-}\left(a d s+\sigma d W_{s}\right)+\sum_{j=1}^{N_{t}} \xi_{\tau_{j}}^{H} X_{\tau_{j}-} U_{j}+L_{t}
$$

where $\xi^{H}$ and $L$ satisfy the conditions of Definition 2.2. Theorem 3.4 of 13 implies the existence and uniqueness of the Föllmer-Schweizer decomposition; thus $\xi^{H}$ is uniquely defined.

We will find $\xi^{H}$ such that $[L, M]_{0 \leq t \leq T}$ is a martingale. We have

$$
\begin{aligned}
{[L, M]_{t}=} & {\left[\int_{0}^{t} \psi_{s} X_{s-}\left(a d s+\sigma d W_{s}\right)+\int_{0}^{t} \varrho_{s} d s+\sum_{j: 0<\tau_{j} \leq t}\left(V_{\tau_{j}}-V_{\tau_{j}-}\right)\right.} \\
& -\int_{0}^{t} \xi_{s}^{H} X_{s-}\left(a d s+\sigma d W_{s}\right)-\sum_{j: 0<\tau_{j} \leq t} \xi_{\tau_{j}}^{H} X_{\tau_{j}-} U_{j}, \sigma \int_{0}^{t} X_{s-} d W_{s} \\
& \left.+\sum_{j: 0<\tau_{j} \leq t} X_{\tau_{j}-} U_{j}-E_{1} \lambda \int_{0}^{t} X_{s-} d s\right] \\
= & \sigma^{2} \int_{0}^{t}\left(\psi_{s}-\xi_{s}^{H}\right) X_{s-}^{2} d s \quad \\
& +\sum_{j: 0<\tau_{j} \leq t}\left(V_{\tau_{j}}-V_{\tau_{j}-}-\xi_{\tau_{j}}^{H} X_{\tau_{j}-} U_{j}\right) X_{\tau_{j}-} U_{j} .
\end{aligned}
$$

In order to apply Lemma 2.1, we show that

$$
\left(V_{\tau_{j}}-V_{\tau_{j}-}-\xi_{\tau_{j}}^{H} X_{\tau_{j}-} U_{j}\right) X_{\tau_{j}-} U_{j}
$$

is a continuous function of two arguments, namely of some left continuous adapted vector process $Y_{t}$ considered at the moment $\tau_{j}$ and of $U_{j}$. Consider

$$
\Phi_{1}(t, y, z)=\mathrm{E}\left[\left\{f\left(y(1+z) \frac{X_{T}}{X_{t}}\right)-f\left(y \frac{X_{T}}{X_{t}}\right)\right\} \frac{\hat{Z}_{T}}{\hat{Z}_{t}}\right] .
$$


Using the Lebesgue dominated convergence theorem one can show that $\Phi_{1}(t, y, z)$ is continuous in $t$ and $y$, since the growth of $f$ is at most polynomial. Recall that $F(t, x)$ is continuous in $t$. The boundedness condition (2.4) in Lemma 2.1 holds, since the growth of $f$ is at most polynomial.

Then

$$
\begin{aligned}
V_{\tau_{j}}-V_{\tau_{j}-} & =F\left(t, X_{t}\right)-\left.F\left(t-, X_{t-}\right)\right|_{t=\tau_{j}}=F\left(t, X_{t}\right)-\left.F\left(t, X_{t-}\right)\right|_{t=\tau_{j}} \\
& =\mathrm{E}\left[f\left(v \frac{X_{T}}{X_{t}}\right) \frac{\hat{Z}_{T}}{\hat{Z}_{t}}\right]-\left.\mathrm{E}\left[f\left(y \frac{X_{T}}{X_{t}}\right) \frac{\hat{Z}_{T}}{\hat{Z}_{t}}\right]\right|_{t=\tau_{j}, y=X_{\tau_{j}-}, v=X_{\tau_{j}}} \\
& =\mathrm{E}\left[f\left(y(1+z) \frac{X_{T}}{X_{t}}\right) \frac{\hat{Z}_{T}}{\hat{Z}_{t}}\right]-\left.\mathrm{E}\left[f\left(y \frac{X_{T}}{X_{t}}\right) \frac{\hat{Z}_{T}}{\hat{Z}_{t}}\right]\right|_{t=\tau_{j}, y=X_{\tau_{j}-, z=U_{j}}} \\
& =\left.\Phi_{1}(t, y, z)\right|_{t=\tau_{j}, y=X_{\tau_{j}-}, z=U_{j}}
\end{aligned}
$$

Now we apply Lemma 2.1 for the vector process $\left(Y_{t}\right)=\left(t, X_{t-}, \xi_{t}^{H}\right)$ and for the function

$$
\Phi\left(y_{1}, y_{2}, y_{3}, z\right)=\Phi_{1}\left(y_{1}, y_{2}, y_{3}, z\right) y_{1} y_{2} \text {. }
$$

This justifies the application of Lemma 2.1 to the sum of terms of the form (3.13).

According to (3.12) and Lemma 2.1, if

$$
\begin{gathered}
\sigma^{2} \int_{0}^{t}\left(\psi_{s}-\xi_{s}^{H}\right) X_{s-}^{2} d s+\lambda \int_{0}^{t} d s \int_{\mathbb{R}} \nu(d z)\left(\Phi_{1}\left(s, X_{s-}, z\right) X_{s-} z-\xi_{s}^{H} X_{s-}^{2} z^{2}\right)=0 \\
\Leftrightarrow \sigma^{2}\left(\psi_{s}-\xi_{s}^{H}\right) X_{s-}^{2}+\lambda \int_{\mathbb{R}} \nu(d z)\left(\Phi_{1}\left(s, X_{s-}, z\right) X_{s-} z-\xi_{s}^{H} X_{s-}^{2} z^{2}\right)=0 \\
\Leftrightarrow \sigma^{2} \psi_{s} X_{s-}^{2}+\lambda X_{s-} \int_{\mathbb{R}} \nu(d z) \Phi_{1}\left(s, X_{s-}, z\right) z=\left(\sigma^{2}+\lambda E_{2}\right) \xi_{s}^{H} X_{s-}^{2}
\end{gathered}
$$

for all $t$, then $[L, M]_{0 \leq t \leq T}$ is a martingale. Thus the process

$$
\xi_{s}^{H}=\frac{1}{\sigma^{2}+\lambda E_{2}}\left(\sigma^{2} \psi_{s}+\frac{\lambda}{X_{s-}} \int_{\mathbb{R}} \nu(d z) \Phi_{1}\left(s, X_{s-}, z\right) z\right)
$$

is left continuous and such that $[L, M]_{0 \leq t \leq T}$ is a martingale; moreover $L$ is orthogonal to $M$.

Let $\tilde{U}$ be a random variable that is independent of all other random variables and whose distribution coincides with that of $U_{j}$. Then

$$
\xi_{t}^{H}=\frac{1}{\sigma^{2}+\lambda E_{2}}\left(\sigma^{2} \frac{\partial F}{\partial x}\left(t, X_{t-}\right)+\frac{\lambda}{X_{t-}} G\left(t, X_{t-}\right)\right),
$$

where

$$
G(t, y)=\mathrm{E}\left[\left\{f\left(y(1+\tilde{U}) \frac{X_{T}}{X_{t}}\right)-f\left(y \frac{X_{T}}{X_{t}}\right)\right\} \frac{\hat{Z}_{T}}{\hat{Z}_{t}} \tilde{U}\right]
$$

by (3.10). The inclusion $\left(\xi_{t}^{H}\right) \in \Theta$ is checked in a standard way.

Further

$$
\begin{aligned}
L_{t}= & V_{t}-V_{0}-\int_{0}^{t} \xi_{s}^{H} d X_{s} \\
= & \int_{0}^{t}\left(\psi_{s}-\xi_{s}^{H}\right) X_{s-}\left(a d s+\sigma d W_{s}\right) \\
& +\int_{0}^{t} \varrho_{s} d s+\sum_{j: 0<\tau_{j} \leq t}\left(V_{\tau_{j}}-V_{\tau_{j}-}-\xi_{\tau_{j}}^{H} X_{\tau_{j}-} U_{j}\right) .
\end{aligned}
$$


Since the Föllmer-Schweizer decomposition holds and is unique, $\left(L_{t}\right)_{0 \leq t \leq T}$ is a martingale.

Lemma 2.1 implies that the process

$$
\begin{aligned}
& \sum_{j: 0<\tau_{j} \leq t}\left(V_{\tau_{j}}-V_{\tau_{j}-}-\xi_{\tau_{j}}^{H} X_{\tau_{j}-} U_{j}\right)-\lambda \int_{0}^{t} d s \int_{\mathbb{R}} \nu(d z)\left(\Phi_{1}\left(s, X_{s-}, z\right)-\xi_{s}^{H} X_{s-} z\right) \\
& =\sum_{j: 0<\tau_{j} \leq t}\left(V_{\tau_{j}}-V_{\tau_{j}-}-\xi_{\tau_{j}}^{H} X_{\tau_{j}-} U_{j}\right)-\lambda \int_{0}^{t} d s \int_{\mathbb{R}} \nu(d z) \Phi_{1}\left(s, X_{s-}, z\right) \\
& \quad+E_{1} \lambda \int_{0}^{t} \xi_{s}^{H} X_{s-} d s
\end{aligned}
$$

is a martingale. Thus

$$
\sum_{j: 0<\tau_{j} \leq t}\left(V_{\tau_{j}}-V_{\tau_{j}-}-\xi_{\tau_{j}}^{H} X_{\tau_{j}-} U_{j}\right)-\lambda \int_{0}^{t} G_{1}\left(s, X_{s-}\right) d s+E_{1} \lambda \int_{0}^{t} \xi_{s}^{H} X_{s-} d s
$$

is a martingale, too, where

$$
G_{1}(t, y)=\mathrm{E}\left[\left\{f\left(y(1+\tilde{U}) \frac{X_{T}}{X_{t}}\right)-f\left(y \frac{X_{T}}{X_{t}}\right)\right\} \frac{\hat{Z}_{T}}{\hat{Z}_{t}}\right]
$$

(recall that the random variable $\tilde{U}$ is independent of other random elements and has the same distribution as $U_{j}$ ).

Hence

$$
\begin{aligned}
L_{t}= & \sigma \int_{0}^{t}\left(\psi_{s}-\xi_{s}^{H}\right) X_{s-} d W_{s}+\sum_{j: 0<\tau_{j} \leq t}\left(V_{\tau_{j}}-V_{\tau_{j}-}-\xi_{\tau_{j}}^{H} X_{\tau_{j}-} U_{j}\right) \\
& -\lambda \int_{0}^{t} G_{1}\left(s, X_{s-}\right) d s+E_{1} \lambda \int_{0}^{t} \xi_{s}^{H} X_{s-} d s .
\end{aligned}
$$

The difference between the right hand sides of (3.15) and (3.17) is a process of bounded variation. Since $\left(L_{t}\right)_{0 \leq t \leq T}$ is a martingale, the latter process is zero almost surely.

Combining the above reasoning we get the following result.

Theorem 3.1. Let the discounted price $\left(X_{t}\right)_{0 \leq t \leq T}$ of a stock satisfy the assumptions of the model (2.1)-(2.2) and let $f: \mathbb{R} \rightarrow \mathbb{R}_{+}$be a continuous function whose growth is at most polynomial. Then the elements $\xi_{t}^{H}$ and $L_{t}^{H}$ of the Föllmer-Schweizer decomposition (2.7) of the contingent claim $H=f\left(X_{T}\right)$ are given by

$$
\begin{aligned}
\xi_{t}^{H}=\frac{1}{\sigma^{2}+\lambda E_{2}} & \left(\sigma^{2} \frac{\partial F}{\partial x}\left(t, X_{t-}\right)+\frac{\lambda}{X_{t-}} G\left(t, X_{t-}\right)\right), \\
L_{t}^{H}=L_{t}=\sigma & \int_{0}^{t}\left(\frac{\partial F}{\partial x}\left(s, X_{s-}\right)-\xi_{s}^{H}\right) X_{s-} d W_{s} \\
& +\sum_{j: 0<\tau_{j} \leq t}\left(V_{\tau_{j}}-V_{\tau_{j}-}-\xi_{\tau_{j}}^{H} X_{\tau_{j}-} U_{j}\right) \\
& -\lambda \int_{0}^{t} G_{1}\left(s, X_{s-}\right) d s+E_{1} \lambda \int_{0}^{t} \xi_{s}^{H} X_{s-} d s
\end{aligned}
$$

where $F, G, G_{1}$ are given by (3.1), (3.14), and (3.16), respectively.

The element $H_{0}$ (the price of $H$ ) is defined by equality (2.11). 


\section{Derivation of the hedging Strategy}

Equality (2.2) of the paper [1] becomes of the following form for our model:

$$
\xi_{t}^{\left(H_{0}\right)}=\xi_{t}^{H}+\alpha_{t}\left(\int_{0}^{t-} \xi_{s}^{H} d X_{s}-\int_{0}^{t-} \xi_{s}^{\left(H_{0}\right)} d X_{s}+L_{t-}\right), \quad 0<t \leq T .
$$

Put $\eta_{t}=\xi_{t}^{\left(H_{0}\right)}-\xi_{t}^{H}$ and $\eta_{0-}=0$. Then

$$
\eta_{t}=\alpha_{t}\left(L_{t-}-\int_{0}^{t-} \eta_{s} d X_{s}\right)
$$

Let $\tilde{\eta}_{t}=\eta_{t} / \alpha_{t}$. It follows from (2.6) that $\alpha_{t}=\rho / X_{t-}$. Now we obtain from (2.1) and (4.1) that

$$
\tilde{\eta}_{t}=L_{t-}-\rho \int_{0}^{t-} \tilde{\eta}_{s}\left(a d s+\sigma d W_{s}\right)-\rho \sum_{j: 0<\tau_{j}<t} \tilde{\eta}_{\tau_{j}} U_{j}=L_{t-}+\int_{0}^{t-} \tilde{\eta}_{s} d Y_{s},
$$

where $Y_{t}=-\rho\left(a t+\sigma W_{t}+\sum_{j: 0<\tau_{j} \leq t} U_{j}\right)$. By $\mathcal{E}(Y)_{t}$ we denote the stochastic exponent of the process $Y_{t}$ such that $\mathcal{E}(Y)_{0}=1$. Then

$$
d \mathcal{E}(Y)_{t}=\mathcal{E}(Y)_{t-} d Y_{t}
$$

(see Theorem 13.5 in [19]). For the right continuous process $\hat{\eta}_{t}=\tilde{\eta}_{t+}$, we obtain the equation

$$
\hat{\eta}_{t}=L_{t}+\int_{0}^{t} \hat{\eta}_{s-} d Y_{s}
$$

Theorem 14.6 in [19] proves the existence and uniqueness of a solution of equation (4.2). We find the solution of (4.2) in the form of

$$
\hat{\eta}_{t}=\zeta_{t} \mathcal{E}(Y)_{t}
$$

where

$$
d \zeta_{t}=\phi_{t} d W_{t}+\beta_{t} d t+d\left(\sum_{j: 0<\tau_{j} \leq t} \gamma_{j}\right)
$$

is an unknown right continuous and $\mathcal{F}_{t}$-adapted process. By Itô's formula,

$$
d \hat{\eta}_{t}=\zeta_{t-} \mathcal{E}(Y)_{t-} d Y_{t}+\mathcal{E}(Y)_{t-} d \zeta_{t}+d[\zeta, \mathcal{E}(Y)]_{t} .
$$

Using (4.2), we get

$$
\mathcal{E}(Y)_{t-} d \zeta_{t}+d[\zeta, \mathcal{E}(Y)]_{t}=d L_{t} .
$$

Now we derive from (3.19), (4.3), and (4.4) that

$$
\begin{aligned}
\phi_{t} \mathcal{E}(Y)_{t-} d W_{t}+\mathcal{E}(Y)_{t-}\left(\beta_{t}-\phi_{t} \sigma \rho\right) d t+d\left(\sum_{j: 0<\tau_{j} \leq t} \mathcal{E}(Y)_{\tau_{j}-} \gamma_{j}\left(1-\rho U_{j}\right)\right) \\
=\sigma\left(\psi_{t}-\xi_{t}^{H}\right) X_{t-} d W_{t}+\lambda\left(E_{1} \xi_{t}^{H} X_{t-}-G_{1}\left(t, X_{t-}\right)\right) d t \\
\quad+d\left(\sum_{j: 0<\tau_{j} \leq t}\left(V_{\tau_{j}}-V_{\tau_{j}-}-\xi_{\tau_{j}}^{H} X_{\tau_{j}-} U_{j}\right)\right) .
\end{aligned}
$$

Therefore

$$
\begin{gathered}
\phi_{t}=\frac{\sigma X_{t-}}{\mathcal{E}(Y)_{t-}}\left(\psi_{t}-\xi_{t}^{H}\right), \\
\beta_{t}=\frac{1}{\mathcal{E}(Y)_{t-}}\left[E_{1} \lambda \xi_{t}^{H} X_{t-}-\lambda G_{1}\left(t, X_{t-}\right)+\sigma^{2} \rho X_{t-}\left(\psi_{t}-\xi_{t}^{H}\right)\right], \\
\gamma_{j}=\frac{V_{\tau_{j}}-V_{\tau_{j}-}-\xi_{\tau_{j}}^{H} X_{\tau_{j}-} U_{j}}{\mathcal{E}(Y)_{\tau_{j}-}\left(1-\rho U_{j}\right)} .
\end{gathered}
$$


Recall that

$$
\eta_{t}=\alpha_{t} \tilde{\eta}_{t}=\frac{\rho}{X_{t-}} \hat{\eta}_{t-}=\frac{\rho}{X_{t-}} \zeta_{t-} \mathcal{E}(Y)_{t-}
$$

Thus

$$
\begin{aligned}
\eta_{t}=\frac{\rho \mathcal{E}(Y)_{t-}}{X_{t-}}\left\{\int_{0}^{t} \frac{\sigma X_{s-}}{\mathcal{E}(Y)_{s-}}\left(\frac{\partial F}{\partial x}\left(s, X_{s-}\right)-\xi_{s}^{H}\right) d W_{s}\right. \\
+\int_{0}^{t} \frac{1}{\mathcal{E}(Y)_{s-}}\left[E_{1} \lambda \xi_{s}^{H} X_{s-}-\lambda G_{1}\left(s, X_{s-}\right)\right. \\
\left.+\sigma^{2} \rho X_{s-}\left(\frac{\partial F}{\partial x}\left(s, X_{s-}\right)-\xi_{s}^{H}\right)\right] d s \\
\left.+\sum_{j: 0<\tau_{j}<t} \frac{V_{\tau_{j}}-V_{\tau_{j}-}-\xi_{\tau_{j}}^{H} X_{\tau_{j}-} U_{j}}{\mathcal{E}(Y)_{\tau_{j}-}\left(1-\rho U_{j}\right)}\right\} .
\end{aligned}
$$

Collecting the above results we prove the following theorem.

Theorem 4.1. Let the assumptions of Theorem 3.1 hold. Then the number of stocks suggested by the minimum variance hedging strategy for the model (2.1)-(2.2) is equal to $\xi_{t}^{\left(H_{0}\right)}=\xi_{t}^{H}+\eta_{t}$, where $\xi_{t}^{H}$ and $\eta_{t}$ are defined by equalities (3.18) and (4.5), respectively.

The price of the contingent claim $H$ in the minimum variance hedging strategy is given by formula (2.11).

If $\rho=0$ (that is, if $X_{t}$ is a martingale), then the result of Theorem 4.1 coincides with the formulas obtained in Section 7.3.4 of [7.

Recall that $\xi_{t}^{\left(H_{0}\right)}$ is equal to the number of stocks (each of them costs $X_{t}$ ) possessed by an investor at time $t$. Then the discounted wealth of the investor portfolio at the moment $t$ equals

$$
V_{t}=H_{0}+\int_{0}^{t} \xi_{s}^{\left(H_{0}\right)} d X_{s}
$$

The strategy is self-financing; thus the discounted capital invested in nonrisky assets at the moment $t$ is equal to

$$
H_{0}+\int_{0}^{t} \xi_{s}^{\left(H_{0}\right)} d X_{s}-\xi_{t}^{\left(H_{0}\right)} X_{t}
$$

\section{Appendix. The proof of the absolute continuity With RESPECT TO LEBESGUe MEASURE}

We prove that the trajectories of the process

$$
\sum_{j: 0<\tau_{j} \leq t} R\left(\tau_{j-1}, \tau_{j}-, X_{\tau_{j-1}}\right)+R\left(\tau_{N_{t}}, t, X_{\tau_{N_{t}}}\right), \quad 0 \leq t \leq T,
$$

are absolutely continuous on $[0, T]$, where $R$ is defined by (3.9) (this result is used above in (3.11) $)$. Since $R\left(\tau_{j}, \tau_{j}, X_{\tau_{j}}\right)=0$, it suffices to show that $R\left(\tau_{j}, t, X_{\tau_{j}}\right)$ is absolutely continuous for $t \in\left[\tau_{j}, \tau_{j+1}\right]$.

For some $K$ and $n$, we have $f(x) \leq K\left(|x|^{n}+1\right), x \in \mathbb{R}$. For simplicity, we assume that $f(x) \leq K|x|^{n}$ (the general case is considered similarly). 
Applying the latter inequality for a fixed $\omega^{N}$, we have

$$
\begin{aligned}
& \bar{F}\left(t, x, \omega^{N}\right) \\
& \leq K \mathrm{E}^{U} \mathrm{E}^{W}\left[\left(x \exp \left\{\left(a-\frac{\sigma^{2}}{2}\right)(T-t)+\sigma\left(W_{T}-W_{t}\right)\right\} \prod_{j: t<\tau_{j}<T}\left(1+U_{j}\right)\right)^{n}\right. \\
& \times\left.\exp \left\{E_{1} \lambda \rho(T-t)-\frac{1}{2} \sigma^{2} \rho^{2}(T-t)-\sigma \rho\left(W_{T}-W_{t}\right)\right\} \prod_{j: t<\tau_{j}<T}\left(1-\rho U_{j}\right)\right] \\
&=K x^{n} \mathrm{E}^{U}\left[\left(\prod_{j: t<\tau_{j}<T}\left(1+U_{j}\right)\right)^{n} \prod_{j: t<\tau_{j}<T}\left(1-\rho U_{j}\right)\right] \\
& \times \mathrm{E}^{W}\left[\exp \left\{\left(a-\frac{\sigma^{2}}{2}\right) n(T-t)+\sigma n\left(W_{T}-W_{t}\right)\right\}\right. \\
&\left.\quad \times \exp \left\{E_{1} \lambda \rho(T-t)-\frac{1}{2} \sigma^{2} \rho^{2}(T-t)-\sigma \rho\left(W_{T}-W_{t}\right)\right\}\right] \\
&=K x^{n}\left[\mathrm{E}^{U}\left(1+U_{j}\right)^{n}\left(1-\rho U_{j}\right)\right]^{N_{T}-N_{t}} M_{1} \leq K x^{n} l^{N_{T}-N_{t}} K_{1} \leq K_{2} x^{n} l^{N_{T}} .
\end{aligned}
$$

The above expectations $\mathrm{E}^{W}$ define a continuous function of $t \in[0, T]$; its maximum is denoted by $K_{1}$. Also

$$
K_{2}=K K_{1}, \quad l=\max \left\{1, \mathrm{E}^{U}\left(1+U_{j}\right)^{n}\left(1-\rho U_{j}\right)\right\} .
$$

Then

$$
\bar{F}\left(\tau_{k}, \bar{X}_{\tau_{k}}^{(j)}, \omega^{N}\right) \leq K_{2}\left(\bar{X}_{\tau_{k}}^{(j)}\right)^{n} l^{N_{T}} .
$$

This inequality together with (3.5) implies that

$$
\begin{aligned}
\left|R\left(s, t, x_{j}\right)\right| & \leq \mathrm{E}^{N}\left|\sum_{k: s<\tau_{k} \leq t}\left[\bar{F}\left(\tau_{k}, \bar{X}_{\tau_{k}}^{(j)}, \omega^{N}\right)-\bar{F}\left(\tau_{k}-, \bar{X}_{\tau_{k}}^{(j)}, \omega^{N}\right)\right]\right| \\
& \leq \mathrm{E}^{N} K_{2} l^{N_{T}}\left(2 \sum_{k: s<\tau_{k} \leq t}\left(\bar{X}_{\tau_{k}}^{(j)}\right)^{n}\right) \\
& \leq 2 K_{2} x_{j}^{n}\left\{\sup _{0 \leq u \leq T} \exp \left\{2\left(a-\frac{\sigma^{2}}{2}\right) u+2 \sigma\left|W_{u}\right|\right\}\right\}^{n} \mathrm{E}^{N}\left\{l^{N_{T}}\left(N_{t}-N_{s}\right)\right\}
\end{aligned}
$$

for $s, t \in\left[b_{j}, c_{j}\right], s<t$. Since the increments of the process $\left(N_{t}\right)$ are independent, we get

$$
\begin{aligned}
\mathrm{E}^{N} & \left\{l^{N_{T}}\left(N_{t}-N_{s}\right)\right\}=\mathrm{E}^{N}\left\{l^{N_{s}}\right\} \mathrm{E}^{N}\left\{l^{N_{t}-N_{s}}\left(N_{t}-N_{s}\right)\right\} \mathrm{E}^{N}\left\{l^{N_{T}-N_{t}}\right\} \\
& =\exp \{(l-1) s \lambda\} l(t-s) \lambda \exp \{(l-1)(t-s) \lambda\} \exp \{(l-1)(T-t) \lambda\} \\
& \leq K_{3}(t-s),
\end{aligned}
$$

where $K_{3}$ is a constant. Thus, for some random variable $K_{4}$,

$$
\left|R\left(s, t, x_{j}\right)\right| \leq K_{4} x_{j}^{n}(t-s)
$$

For a fixed $\omega \in \Omega$ and $s, t \in\left[\tau_{j}, \tau_{j+1}\right], s<t$, the definition of $R$ implies that

$$
\left|R\left(\tau_{j}, t, X_{\tau_{j}}\right)-R\left(\tau_{j}, s, X_{\tau_{j}}\right)\right|=\left|R\left(s, t, X_{\tau_{j}}\right)\right| \leq K_{4} X_{\tau_{j}}^{n}(t-s) .
$$

This completes the proof of the absolute continuity. 


\section{BIBLIOGRAPHY}

1. M. Schweizer, Approximating random variables by stochastic integrals, Ann. Probab. 22 (1994), 1536-1575. MR1303653 (95j:60069)

2. H. Pham, T. Rheinländer, and M. Schweizer, Mean-variance hedging for continuous processes: New proofs and examples, Finance Stoch. 2 (1998), 173-198. MR.1806102 (2001h:91049)

3. Ch. Hou and I. Karatzas, Least-squares approximation of random variables by stochastic integrals, Adv. Stud. Pure Math., vol. 41, Math. Soc. Japan, Tokyo, 2004, pp. 141-166. MR2083708 (2005g:60105)

4. J. P. Ansel and C. Stricker, Lois de martingale, densités et décomposition de FöllmerSchweizer, Ann. Inst. H. Poincaré Probab. Statist. 28 (1992), 375-392. MR.1183992 (94d:60069)

5. R. Merton, Option pricing when underlying stock returns are discontinuous, J. Financial Economics 3 (1976), 125-144.

6. R. Cont and P. Tankov, Financial Modelling with Jump Processes, Chapman and Hall, London, 2004. MR 2042661 (2004m:91004)

7. D. Lamberton and B. Lapeyre, Introduction to Stochastic Calculus Applied to Finance, Chapman and Hall, London, 1996. MR.1422250 (98b:90018)

8. F. Hubalek, J. Kallsen, and L. Krawczyk, Variance-optimal hedging for processes with stationary independent increments, Ann. Appl. Probab. 16 (2006), no. 2, 853-885. MR2244435 (2007k:60205)

9. M. Mania, M. Santacroce, and R. Tevzadze, A semimartingale BSDE related to the minimal entropy martingale measure, Finance Stoch. 7 (2003), 385-402. MR1994915 (2004f:91080)

10. M. Schweizer, Option hedging for semimartingales, Stoch. Process. Appl. 37 (1991), 339-363. MR.1102880 (92c:90025)

11. V. M. Radchenko, Minimal risk strategy for processes with jumps, Applied Statistics. Actuarial and Finance Mathematics 1-2 (2003), 55-68. (Ukrainian)

12. V. N. Radchenko, Minimum variance hedging in a model with jumps at nonrandom moments of time, Teor. Veroyatn. Primenen. 51 (2006), no. 3, 608-618; English transl. in Theory Probab. Appl. 51 (2007), no. 3, 536-545. MR2325550(2008e:60210)

13. P. Monat and C. Stricker, Föllmer-Schweizer decomposition and mean-variance hedging for general claims, Ann. Probab. 23 (1995), 605-628. MR.1334163 (97m:60065)

14. H. Föllmer and M. Schweizer, Hedging of contingent claims under incomplete information, Applied Stochastic Analysis (M. H. A. Davis and R. J. Elliott, eds.), Stochastics Monogr., vol. 5, Gordon and Breach, London-New York, 1991, pp. 389-414. MR.1108430 (92g:90029)

15. M. Schweizer, On the minimal martingale measure and the Föllmer-Schweizer decomposition, Stoch. Anal. Appl. 13 (1995), 573-599. MR1353193 (96h:60080)

16. M. Schweizer, Approximation pricing and the variance-optimal martingale measure, Ann. Probab. 24 (1996), 206-236. MR1387633 (97c:60120)

17. X. L. Zhang, Valuation of American options in a jump-diffusion model, Numerical Methods in Finance, Publications of the Newton Institute, vol. 13, Cambridge University Press, Cambridge, 1997, pp. 93-114. MR.1470511 (98e:90032)

18. A. N. Shiryaev, Essentials of Stochastic Finance. Facts, Models, Theory, Fazis, Moscow, 1998; English transl., World Scientific Publishing Co., Inc., River Edge, NJ, 1999. MR1695318 (2000e:91085)

19. R. J. Elliott, Stochastic Calculus and Applications, Springer-Verlag, New York, 1982. MR 678919 (85b:60059)

20. M. Musiela and M. Rutkowski, Martingale Methods in Financial Modelling, Applications of Mathematics, vol. 36, Springer, Berlin, 1997. MR1474500 (98i:90014)

Department of Mathematical Analysis, Faculty for Mechanics and Mathematics, National Taras Shevchenko University, Academician Glushkov Avenue 6, Kyiv 03127, Ukraine

E-mail address: vradchenko@univ.kiev.ua

Received 7/MAY/2007

Translated by S. KVASKO 